\title{
Acquired Factor V Inhibitor Developing after Treatment with Dabigatran Etexilate Methanesulfonate: A Case Report and Review of the Literature
}

\author{
Yasunobu Sekiguchi - Hitomi Yoshikawa - Asami Shimada - Hidenori Imai · \\ Mutsumi Wakabayashi $\cdot$ Keiji Sugimoto • Noriko Nakamura • Tomohiro Sawada • \\ Tetsuo Ichinose $\cdot$ Dai Ozaki $\cdot$ Norio Komatsu $\cdot$ Masaaki Noguchi
}

Received: 19 December 2013/Accepted: 15 February 2014/Published online: 6 March 2014

(C) Indian Society of Haematology \& Transfusion Medicine 2014

\begin{abstract}
A 90-year-old man presented with subcutaneous ecchymoses. He had been under treatment with dabigatran etexilate methanesulfonate (DEM). Prolonged APTT and decreased PT was developed 2 months after the start of DEM, more prolonged 6 months later. DEM was discontinued, the coagulopathy did not improve. Factor $\mathrm{V}$ activity was decreased, along with appearance of coagulation factor $\mathrm{V}$ inhibitor (FVI). He did not have antiphospholipid syndrome or malignancy. He was diagnosed as having acquired FVI caused by DEM. Steroid pulse therapy was effective. There have been 74 reported cases of AFVI induced by drug treatment, but none after treatment with DEM.
\end{abstract}

Keywords Dabigatran etexilate methanesulfonate . Acquired factor $\mathrm{V}$ inhibitor - Coagulation factor $\mathrm{V}$ deficiency $\cdot$ Steroid pulse

Y. Sekiguchi $(\bowtie) \cdot$ H. Yoshikawa - A. Shimada · H. Imai ·

M. Wakabayashi · K. Sugimoto · N. Komatsu · M. Noguchi Department of Hematology, Juntendo University Urayasu Hospital, 1-1, 2-Chome, Tomioka, Urayasu, Chiba, Japan e-mail: yasu_sek@juntendo-urayasu.jp

N. Nakamura · T. Sawada

Department of Laboratory Medicine, Juntendo University

Urayasu Hospital, Urayasu, Chiba, Japan

T. Ichinose

Department of Cardiology, Kohnodai Hospital, National Center for Global Health and Medicine, Ichikawa, Japan

D. Ozaki

Department of Cardiology, Juntendo University Urayasu Hospital, Urayasu, Chiba, Japan

\section{Introduction}

Clinical symptoms of acquired factor V inhibitor (AFVI) are varied, ranging from asymptomatic to hemorrhage and thrombotic tendency [1]. Some of the consequences may be fatal [2], underscoring the need for prompt diagnosis and treatment. In addition, various causes of AFVI have been reported, and approximately $30 \%$ of cases are idiopathic [3]. To the best of our knowledge, there have been no reports of AFVI caused by treatment with dabigatran etexilate methanesulfonate (DEM). DEM was first approved for use in the field of orthopedic surgery for the primary prevention of venous thromboembolic events in Europe in March 2008. Thereafter, it was approved for use in the US in October 2010, in Japan in January 2011, and in Europe in August 2011, as an agent for reducing the risk of stroke and systemic embolism in patients with nonvalvular atrial fibrillation. Thus, DEM is a relatively new drug. DEM suppresses the formation of fibrin from fibrinogen through direct and selective inhibition of thrombin activity, thereby exerting anticoagulant and antithrombotic effects [4]. Use of DEM is expected to increase in the future, because of its advantages, such as availability as a formulation for oral use and better controllability of blood coagulability than warfarin. Here, we report a patient who developed AFVI following treatment with this drug, in whom careful treatment was considered necessary.

\section{Case Report}

A 90-year-old man presented with generalized ecchymoses. His medical history included sleep apnea, gastric ulcer, and skin photosensitivity. He had no significant family history. He was under treatment with telmisartan, 
Table 1 Laboratory findings

\begin{tabular}{|c|c|}
\hline \multicolumn{2}{|l|}{$\mathrm{CBC}$} \\
\hline WBC & $4,400 / \mu 1$ \\
\hline $\mathrm{RBC}$ & $433 \times 10^{4} / \mu 1$ \\
\hline $\mathrm{Hb}$ & $12.5 \mathrm{~g} / \mathrm{dl} \downarrow(14.0-18.0)^{*}$ \\
\hline $\mathrm{Ht}$ & $39.1 \% \downarrow(40.0-48.0)^{*}$ \\
\hline $\mathrm{MCV}$ & $90.3 \mathrm{fl}$ \\
\hline $\mathrm{MCH}$ & $28.9 \mathrm{pg}$ \\
\hline Plt & $19.1 \times 10^{4} / \mu 1$ \\
\hline \multicolumn{2}{|l|}{ Coagulation } \\
\hline PT & $74.2 \mathrm{~s} \uparrow(10.0-13.0)^{*}$ \\
\hline PT activity & $<20 \% \downarrow(70-100)^{*}$ \\
\hline PT ratio & $5.89 \uparrow(0.9-1.1)^{*}$ \\
\hline PT-INR & $5.11 \uparrow(0.9-1.1)^{*}$ \\
\hline APTT & $140.3 \mathrm{~s} \uparrow(28-40)^{*}$ \\
\hline Fbg & $375.0 \mathrm{mg} / \mathrm{dl}$ \\
\hline D-D & $1.1 \mu \mathrm{g} / \mathrm{ml} \uparrow(0.0-0.5)^{*}$ \\
\hline Bleeding time & $2 \min$ \\
\hline \multicolumn{2}{|l|}{ Biochemistry } \\
\hline AST & $26 \mathrm{IU} / 1$ \\
\hline ALT & $10 \mathrm{IU} / 1$ \\
\hline LDH & $276 \mathrm{IU} / 1 \uparrow(119-229)^{*}$ \\
\hline$\gamma$-GTP & $17 \mathrm{IU} / 1$ \\
\hline T-Bil & $0.5 \mathrm{mg} / \mathrm{dl}$ \\
\hline BUN & $30 \mathrm{mg} / \mathrm{dl} \uparrow *$ \\
\hline $\mathrm{Cr}$ & $1.31 \mathrm{mg} / \mathrm{dl} \uparrow(0.61-1.04)^{*}$ \\
\hline \multicolumn{2}{|l|}{ Immuno-serological findings } \\
\hline $\mathrm{IgG}$ & $2,508 \mathrm{mg} / \mathrm{dl} \uparrow(870-1,700)^{*}$ \\
\hline $\operatorname{Ig} \mathrm{A}$ & $585 \mathrm{mg} / \mathrm{dl} \uparrow(110-410)^{*}$ \\
\hline $\operatorname{IgM}$ & $55 \mathrm{mg} / \mathrm{dl}$ \\
\hline Antinuclear antibody & $\times 160 \uparrow(0-40)^{*}$ \\
\hline ds-DNA IgG & $<10$ \\
\hline Anticardiolipin IgG & Negative \\
\hline Anti-CL $\beta 2 \mathrm{GP} 1$ antibody & Negative \\
\hline Lupus AC (dRVVT) & Negative \\
\hline \multicolumn{2}{|l|}{ Tumor marker } \\
\hline AFP & $3.7 \mathrm{ng} / \mathrm{ml}$ \\
\hline CEA & $23.3 \mathrm{ng} / \mathrm{ml} \uparrow(0.0-6.5)^{*}$ \\
\hline CA19-9 & $26.4 \mathrm{U} / \mathrm{ml}$ \\
\hline PSA & $0.761 \mathrm{ng} / \mathrm{ml}$ \\
\hline $\mathrm{SCC}$ & $4.6 \mathrm{ng} / \mathrm{ml} \uparrow(0.0-1.5)^{*}$ \\
\hline PIVKA-2 & 27 mAU/ml \\
\hline \multicolumn{2}{|l|}{ Coagulation factor assay } \\
\hline Factor II & $50 \% \downarrow(75-135)^{*}$ \\
\hline Factor V & $\leq 3 \% \downarrow(70-135)^{*}$ \\
\hline Factor VII & $63 \% \downarrow(75-140)^{*}$ \\
\hline Factor VIII & $79 \%(60-150)$ \\
\hline Factor IX & $77 \%(70-130)$ \\
\hline Factor X & $65 \% \downarrow(70-130)^{*}$ \\
\hline Factor XI & $48 \% \downarrow(75-145)^{*}$ \\
\hline Factor XII & $65 \%(50-150)$ \\
\hline
\end{tabular}

Table 1 continued

\begin{tabular}{ll} 
Factor XIII & $123 \%(70-140)$ \\
Factor II inhibitor & Negative \\
Factor V inhibitor & $4 \mathrm{BU} / \mathrm{ml} \uparrow *$ \\
Factor VII inhibitor & Negative \\
Factor X inhibitor & Negative \\
\hline
\end{tabular}

$* \uparrow$ and $\downarrow$ indicate values higher and lower than normal ranges, respectively. Normal ranges are shown in parentheses

benidipine hydrochloride, furosemide, verapamil hydrochloride and aspirin for thoracic aortic aneurysm, aortic regurgitation (grade II), mitral regurgitation (grade I), hypertension and atrial fibrillation from a local hospital. Multiple cerebral microinfarctions developed, and his medication was changed from aspirin to DEM at $220 \mathrm{mg} /$ day in October 2012. At this time, his activated partial thromboplastin time (APTT) $(33.7 \mathrm{~s}$; reference value, $30.2 \mathrm{~s})$ and prothrombin time (PT) (89.5\%; reference value $70-100 \%$ ) were within normal range. In early December 2012, the APTT became prolonged to $61.6 \mathrm{~s}$, and the dose of DEM was reduced to $150 \mathrm{mg} /$ day. In late December, the APTT was well controlled (49.3 s), and the drug was continued at the same dose. In early April 2013, DEM was discontinued because of marked prolongation of the APTT to $134.9 \mathrm{~s}$ and reduction of the PT to less than $20 \%$. Around this time, ecchymoses and eruptions appeared over the whole body. In mid-May, approximately a month and a half after the discontinuation of DEM, further decrease of the blood coagulability was noted (APTT, $140.2 \mathrm{~s}$ ), and the patient was admitted to a local hospital.

At the time of admission to the local hospital, the patient had a body height of $151 \mathrm{~cm}$ and body weight of $65.4 \mathrm{~kg}$, body temperature of $36.5^{\circ} \mathrm{C}$, blood pressure of $150 / 90 \mathrm{mmHg}$, (irregular) pulse of $100 / \mathrm{min}$, clear consciousness, no conjunctival pallor, no icterus, no hepatosplenomegaly, and no abnormal neurological findings. There were no palpable superficial lymph nodes. Eruptions and ecchymoses with itching were seen on the scalp, face, chest, abdomen, back, and the upper extremities of both sides.

The laboratory findings at the time of admission are shown in Table 1.

His clinical course after admission is shown in Fig. 1. Skin biopsy from the erythematous areas on the face and dorsum of the hand led to a diagnosis of DEM-induced photodermatitis. A drug lymphocyte stimulation test was negative for DEM. The patient was treated with oral antiallergic agents and topical steroids, however, his condition did not improve. In mid-May 2013, the patient's condition deteriorated further, with an APTT of $156.1 \mathrm{~s}$ and PT of less than $20 \%$. Measurement of the coagulation factor 

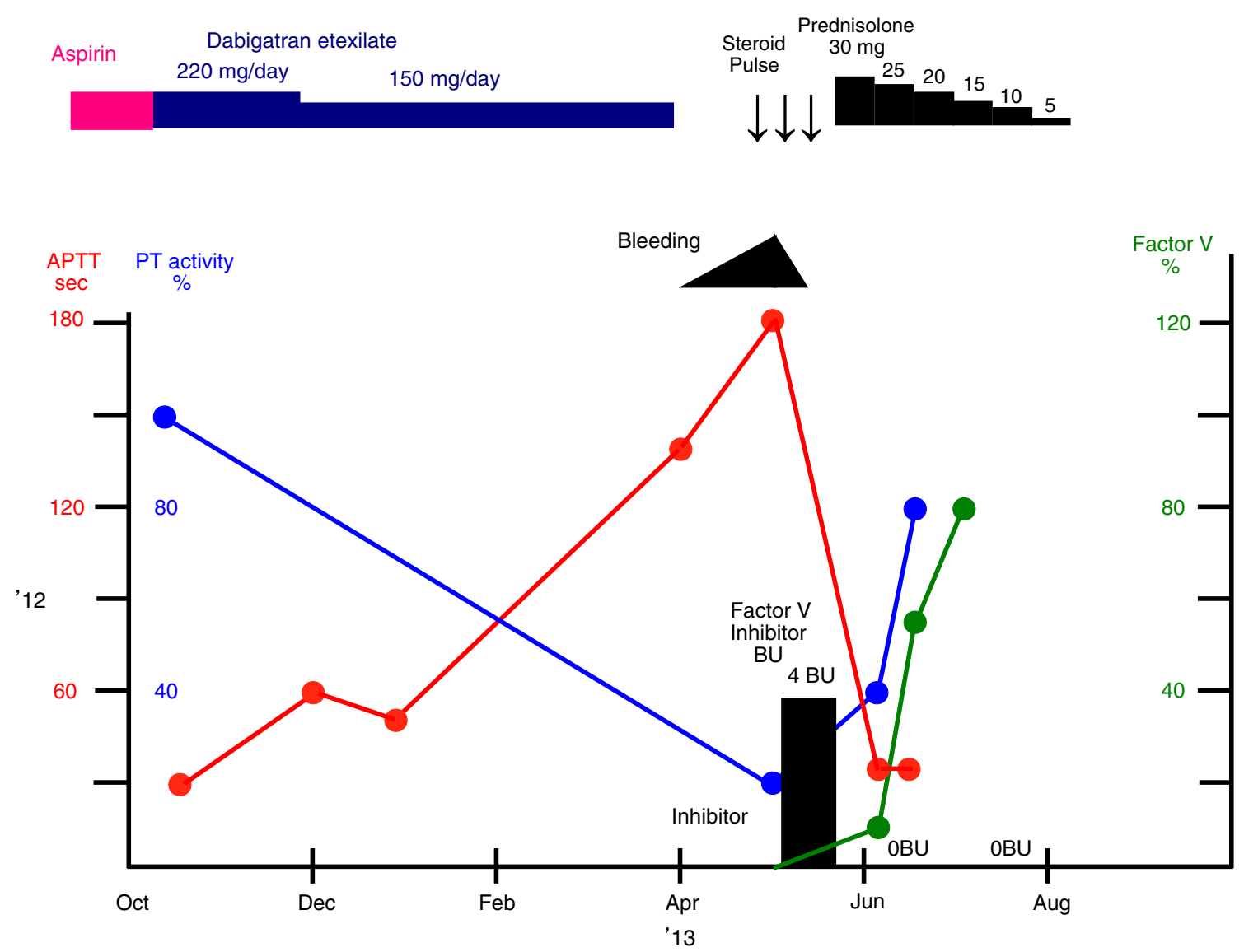

Fig. 1 Clinical course. In October 2012, the medication was changed from aspirin to dabigatran etexilate methanesulfonate (DEM). In early April 2013, because of marked prolongation of the activated partial thromboplastin time (APTT) and decrease of the prothrombin time (PT). In mid-May, he had developed a bleeding tendency. The coagulation factor $\mathrm{V}$ activity decreased again to $\leq 3 \%$ and the test for coagulation factor $\mathrm{V}$ inhibitor activity was positive. The patient was

profile revealed markedly decreased factor $\mathrm{V}$ activity to $\leq 3 \%$ (reference value $70-135 \%$ ) and somewhat reduced activities of factors II, VII, X and XI. There were no abnormal changes of any other coagulation factors. The test for coagulation factor $\mathrm{V}$ inhibitor was positive (4 Bethesda $\mathrm{U} / \mathrm{mL}$ ), while the tests for coagulation inhibitors of factors II, VII, X and XI, which showed reduced activities, were negative. The patient was unlikely to have antiphospholipid syndrome, because of the absence of thrombosis, negative test result for anticardiolipin $\mathrm{IgG}$, and negative test result for anti-CL $32 \mathrm{GP} 1$ antibodies. Based on the above, he was diagnosed as having AFVI activity caused by DEM. In late May, the patient's condition deteriorated further, with an APTT of $\geq 180 \mathrm{~s}$ and PT of $<20 \%$, and ecchymoses appeared over the whole body, being most pronounced on the abdominal wall. Therefore, steroid pulse therapy was administered, followed by continuation of treatment with diagnosed as having acquired factor $\mathrm{V}$ inhibitor (AFVI) induced by DEM. Steroid pulse therapy was administered, following which the coagulation factor $\mathrm{V}$ activity fell within normal range, the test for factor $\mathrm{V}$ inhibitor became negative. The patient was received treatment with prednisolone, which was gradually tapered in dose and finally discontinued. The AFVI has not recurred to date

prednisolone at a daily dose of $30 \mathrm{mg} /$ day. In early June, the patient was referred to our department.

At the time of referral, the test for coagulation factor $\mathrm{V}$ inhibitor was negative and the factor $\mathrm{V}$ activity had improved to $12 \%$. The bleeding tendency had also disappeared. In late June, the dose of prednisolone was gradually reduced to $20 \mathrm{mg} / \mathrm{day}$. Because coagulation factor $\mathrm{V}$ inhibitor remained negative and the factor $\mathrm{V}$ activity and blood coagulability fell within normal range, treatment was started with warfarin. In early July, the patient was discharged and received outpatient treatment with prednisolone, the dose of which was gradually tapered to $5 \mathrm{mg} /$ day. The clinical course remained favorable with negative coagulation factor $\mathrm{V}$ inhibitor activity and factor $\mathrm{V}$ activity within normal range. In addition, slight increases of the serum carcinoembryonic antigen (CEA) $(23.3 \mathrm{ng} /$ $\mathrm{mL}$ ) and squamous cell carcinoma-related antigen (SCC) 
Table 2 Summary of reports of 74 cases of drug-induced acquired factor V inhibitor (AFVI) activity (modified from Ref. [6])

\begin{tabular}{|c|c|c|c|c|}
\hline Age & $\mathrm{M} / \mathrm{F}$ & Drug & Treatment & Results \\
\hline $66.2(16-85)$ & $24 / 9$ & $\begin{array}{l}\beta \text {-lactamase }(n=18) \\
\text { AGS }(n=12) \\
\operatorname{CPFX}(n=6) \\
\operatorname{CPS}(n=4) \\
\text { Tetracycline }(n=2) \\
\text { Warfarin }(n=2) \\
\text { CAF }(n=1) \\
\text { Antibiotics, details unknown }(n=1)\end{array}$ & $\begin{array}{l}\text { Steroid }(n=21) \\
\text { FFP }(n=17) \\
\text { CPA }(n=9) \\
\text { IVIG }(n=7) \\
\text { PLT }(n=7) \\
\text { VK }(n=6) \\
\text { Nothing }(n=3) \\
\text { AZT }(n=3) \\
\text { PCC }(n=3) \\
\operatorname{CsA~}(n=2) \\
\text { AF }(n=2) \\
\operatorname{CP}(n=2) \\
\operatorname{rFVIIa~}(n=2) \\
\operatorname{RTX}(n=2) \\
\operatorname{PEX~}(n=1) \\
\operatorname{SDFP}(n=1)\end{array}$ & $\begin{array}{l}\text { Death }(n=5) \text { (hemorrhage: } n=4 \text {, cause unknown: } n=1) \\
\text { Inhibitor persistence }(n=2) \\
\text { Mean persistence time: } 12.5 \mathrm{M}(6-19 \mathrm{M})\end{array}$ \\
\hline
\end{tabular}

$M / F$ male to female, $A G S$ aminoglycoside, $C P F X$ ciprofloxacin, $C P S$ cephalosporin, $C A F$ chloramphenicol, $F F P$ fresh frozen plasma, $C P A$ cyclophosphamide, IVIG intravenous injection of immunoglobulin, PLT platelet concentrates, VK vitamin K, AZT azathioprine, $P C C$ prothrombin complex concentrates, $C s A$ cyclosporine, $A F$ antifirinolytic agents, $C P$ cryoprecipitates, $r F V I I a$ recombinant factor VII activated, $R T X$ rituximab, $P E X$ plasma exchange, $S D F P$ solvent/detergent-treated frozen plasma, $W$ week, $M$ month

(4.6 ng/mL) were observed, however, whole-body contrast-enhanced CT revealed no malignancies. Upper and lower gastrointestinal endoscopic examinations revealed no abnormalities.

\section{Discussion}

Mild coagulopathy developed approximately 2 months after the start of treatment with DEM and had progressed until 6 months after the start of treatment. The blood coagulability did not improve even after discontinuation of the DEM, and the patient was found to have factor $\mathrm{V}$ inhibitor in the serum, which led to the diagnosis of AFVI activity caused by DEM.

AFVI was first reported in 1955 [5], and approximately 150 cases have been reported to date [3, 5-7]; however, most of the case reports are only descriptive. AFVI is a rare condition, occurring at an incidence of 0.09/100,000 [3] to $0.29 / 1,000,000$ [6] per year. However, a significant percentage are asymptomatic carriers [1], and the actual incidence is speculated to be higher. The clinical symptoms of AFVI vary, ranging from asymptomatic to fatal hemorrhage and thrombotic tendency [1]; the case fatality rate has been reported to be in the range of 14-31\% [2, 7], underscoring the importance of prompt diagnosis and treatment.
One published report provides a summary of 74 case reports of AFVI with meaningful data [7]. Of these, 33 cases in which the AFVI was considered to be caused by drugs are listed in Table 2. The patients were relatively old, with a mean age of 66.2 years, and AFVI was more common in men, with a male/female ratio of 24:9. All causative agents were antibiotics, with $\beta$-lactamases being the most commonly implicated causative agent (18 cases), followed by aminoglycosides (12 cases). The present patient was not treated with antibiotics, and DEM was strongly suspected as the causative agent. According to the aforementioned report, steroids (12 cases) and fresh frozen plasma (FFP) (17 cases) are the most common therapeutic agents for AFVI, followed by cyclophosphamide (CPA), intravenous injection of immunoglobulin (IVIG) and platelet transfusion; steroids were effective in the present patient. Of the 74 reported cases, 26 achieved remission in an average of 8.5 weeks, suggesting that the prognosis of AFVI is relatively good. However, 5 patients died ( 4 died of hemorrhage, and 1 died of unknown cause) and 2 patients had persistent factor $V$ inhibitory in the serum, indicating the need for careful treatment.

The major causes of AFVI other than drugs include surgery, infections, malignancies, autoimmune diseases, and organ transplantation. Blood transfusion, fibrin preparations, bovine thrombin, etc., have also been reported as other causes of AFVI, but these were not used in the present patient. AFVI is idiopathic in $21 \%$ [7] to $30 \%$ [3] 
of the cases. The pathogenic mechanism of AFVI due to DEM is unknown, but if coagulopathy is observed during treatment with DEM, it will be necessary to measure the factor V activity.

No standard treatment for AFVI has been established, however, immunosuppressive agents such as steroids are highly effective [3, 7, 8]. There have been reports of patients with AFVI improving spontaneously without treatment $[3,7,8]$ and treatment is not necessary for asymptomatic patients [9]. However, some patients have fatal hemorrhage $[2,3,7]$, and it is difficult to determine which patients may be indicated for treatment. The present patient showed markedly reduced blood coagulability, with an APTT of $\geq 180 \mathrm{~s}$ and PT of $<20 \%$, and showed a bleeding tendency. Therefore, steroid pulse therapy was administered, and the patient's life was saved. In early June, the factor $\mathrm{V}$ inhibitor disappeared, factor $\mathrm{V}$ activity increased, the blood coagulability fell to within normal range, and the bleeding tendency also disappeared. Therefore, treatment with warfarin was initiated.

There have been 2 reported cases of AFVI after treatment with warfarin $[2,10]$, and it is considered that careful follow-up with periodic measurement of not only the blood coagulability, but also the factor $\mathrm{V}$ activity and factor $\mathrm{V}$ inhibitor is necessary in the future.

There have been no reports of AFVI caused by DEM, and the underlying pathogenic mechanisms, clinical symptoms, treatment and prognosis are unknown. In the future, further analysis will be necessary on a larger number of cases.

In general, the prognosis of AFVI depends on the underlying disease inducing the AFVI [7, 11], and it is important to screen the patients for malignancies [11]. In the present patient, a detailed whole-body examination revealed no malignancies. Prevention of fatal hemorrhage by early detection of AFVI and management of the underlying diseases are considered important for a better prognosis.

\section{References}

1. Ortel TL (1999) Clinical and laboratory manifestations of antifactor V antibodies. J Lab Clin Med 133:326-334

2. Gartrell B (2011) Acquired factor V inhibitor complicating warfarin therapy. Am J Hematol 86:710-712

3. Ang AL, Kuperan P, Ng CH, Ng HJ (2009) Acquired factor V inhibitor. A problem-based systematic review. Thromb Haemost 101:852-859

4. Wienen W, Stassen JM, Priepke H, Ries UJ, Hauel N (2007) Invitro profile and ex vivo anticoagulant activity of the direct thrombin inhibitor dabigatran and its orally active prodrug, dabigatran etexilate. Thromb Haemost 98:155-162

5. Horder MH (1955) Isolated factor V deficiency caused by a specific inhibitor (in German). Acta Haematol 13:235-241

6. Favaloro EJ, Posen J, Ramakrishna R, Soltani S, McRae S, Just S et al (2004) Factor V inhibitors: rare or not so uncommon? A multi-laboratory investigation. Blood Coagul Fibrinolysis 15: 637-647

7. Franchini M, Lippi G (2011) Acquired factor V inhibitors: a systematic review. J Thromb Thrombolysis 31:449-457

8. Streiff MB, Ness PM (2002) Acquired FV inhibitors: a needless iatrogenic complication of bovine thrombin exposure. Transfusion 42:18-26

9. Shanmugam H, Jayaranee S, Eow GI (2011) A case report of an acquired inhibitor to coagulation factor V. Haemophilia 17: $168-170$

10. Nozu T, Mita H, Okumura T (2010) Acquired factor V inhibitor in a patient with a mechanical aortic valve under warfarin therapy. Intern Med 49:2229-2233

11. Endo H, Kawauchi K, Tomimatsu M, Iga D, Ogasawara T, Yasuyama $\mathrm{M}$ et al (2007) Acquired factor $\mathrm{V}$ inhibitor responsive to corticosteroids in a patient with double cancers. Intern Med $46: 621-625$ 